\title{
ARTICLE
}

\section{Development of reconstruction algorithm for activation sample measurement using gamma camera}

\author{
Jae Yong Lee ${ }^{\mathrm{a}}$, Do Hyun Kimª, Che Wook Yima, Jae Hyun Kimª, Myeong Hyeon Woo ${ }^{\mathrm{a}}$, Quang Huy Khuat ${ }^{\mathrm{a}}$, \\ Chang Ho Shin ${ }^{\mathrm{a}}$, Jong Kyung Kim${ }^{\mathrm{a}}$, Bo Kyun $\mathrm{Seo}^{\mathrm{b}}$ and Ji Seok Kim ${ }^{\mathrm{c}}$ \\ ${ }^{a}$ Department of Nuclear Engineering, Hanyang University, 222 Wangsimni-ro, Seongdong-gu, Seoul, Republic of Korea; ${ }^{b}$ Korea \\ Institute of Nuclear Safety, 62 Gwahak-ro, Yuseong-gu, Daejeon, Republic of Korea; ${ }^{c}$ Korea Atomic Energy Research Institute, \\ 111 Daedeok-daero 989beon-gil, Yuseong-gu, Daejeon, 34057, Republic of Korea
}

In a facility using neutron, it is important to figure out the spatial distribution of the neutron beam. To get neutron spatial distributions, multiple activation foils are used in the activation foil method. To measure the radioactivity of foils efficiently, a novel measurement system with gamma camera was proposed. The radioactivity of foils was obtained as the image form in the system. To determine the radioactivity of each foil, reconstruction algorithm was necessary. In this study, a novel reconstruction algorithm suitable to the proposed system was developed. The algorithm was validated by solving two problems. By utilizing the developed algorithm, accurate determination of radioactivity of multiple foils was possible. It is expected that the developed algorithm could be used for the efficient measurement of neutron beam distribution.

Keywords: neutron spatial distribution; neutron activation foil; image reconstruction algorithm

\section{Introduction}

The neutron spatial distribution is one of the important characteristics of the neutron beam facility. There are several methods to measure the spatial distribution of the neutron beam. The imaging plate method is one of the conventional methods [1]. After exposing films in neutron beam field, images are obtained. In this method, metal sheets, and collimators are utilized to remove background signals. Imaging plate have been used in neutron radiography field as high contrast and clear images can be obtained. Another conventional measurement method is the activation foil method. In this method, the neutron distribution is determined by measuring radioactivity of foils after exposing them in the neutron beam field. Foils can be fabricated in small dimensions. As the installation of small foils is easy, the activation foil method has huge applicability. Because of this advantage, the activation foil method is widely used [2]. This study is focused on the improvement of the activation foil method.

To get spatial neutron distribution with the activation foil method, multiple foils are used. Measuring the radioactivity of foils one by one is an inefficient and time-consuming work. For the efficient measurement, a novel system to measure the radioactivity of foils at the same time was proposed. In this measurement system, an array of foils was utilized. The radioactivity of foils

*Corresponding author. Email: jkkim1@hanyang.ac.kr was acquired as an image form. The image was composed of overlapped signals from the array of foils. The radioactivity of each foil is obtained from the image by use of the reconstruction algorithm. The aim of this study was to develop a numerical iterative reconstruction algorithm for the proposed measurement system.

\section{The proposed detection system}

\subsection{The description of the detection system}

A Charge-Coupled Device (CCD) gamma camera and a piece of $\mathrm{CWO}\left(\mathrm{CdWO}_{4}\right)$ scintillator comprised the proposed system [3]. Figure 1 illustrates the structure of the measurement system. Radioactive foils were positioned under the scintillator. Radiations from

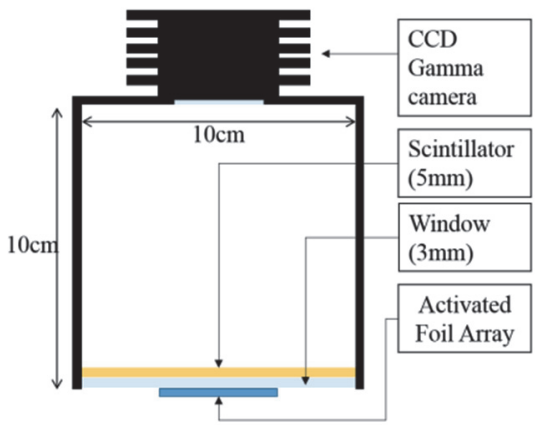

Figure 1. The illustration of the proposed neutron spatial distribution detecting system. 
activated foils were absorbed in the scintillator and photons were emitted from the scintillator. The CCD gamma camera received these photons and an image was obtained.

\subsection{The necessity of the reconstruction algorithm}

Photons emitted from the activated foils are isotropic. The image formed in the CCD gamma camera was composed of overlapped signals from multiple foils. To determine the radioactivity of each foil, it was necessary to separate appropriate signals from overlapped signals in the image. It is a kind of inverse problem. The solution could be obtained by inversion matrix calculation. However, the mathematical value obtained by the simple inversion calculation could be an inappropriate solution because of the observational error in the system. Therefore, to obtain the appropriate solution, a proper reconstruction algorithm was required.

\section{Material and method}

\subsection{Description of the algorithm}

The proposed detection system can be described in a matrix equation as given in Eq. (1).

$$
S T=A
$$

Matrix $S$ represents the radiation source matrix in the radioactive foil array, matrix $A$ describes image formed in the CCD gamma camera. Matrix $T$ contains the contributions of the source matrix to the image matrix. The element of $T$ matrix, $t_{i, j}$ is defined as the contribution of $i$ th element of matrix $S$ to $j$ th element of matrix $A$. Matrices $A$ and $T$ were named as image matrix, and contribution matrix, respectively. The aim was to reconstruct source matrix from image matrix $A$.

If $T$ is non-singular, the Eq. (1) can be solved using inverse matrix. However, there are observational errors in the obtained image. The simple inverse matrix calculation could bring an unacceptable solution. To avoid this problem, an iterative method was used.

The developed method for reconstructing matrix $S$ is presented as follows:

$$
A^{n}=A^{0}-\left(\sum_{i=1}^{n} B^{i}\right) T
$$

where $B$ is a guessed source matrix, and $n$ is the iteration number. The main idea of this method is to choose the peak value of the image matrix as the guessed source matrix in each iteration. This is because the source is likely to be in the strongest signal position in the image matrix. After removing the contribution of the strongest source in the image matrix, the image matrix of the next iteration was obtained. Eq. (1) can be rearranged as given in Eq. (3).

$$
A-S T=O
$$

By comparing Eq. (2) and Eq. (3), an assumption to reconstruct source matrix was made. The assumption was that the image matrix $A^{n}$ was similar to the zero matrix, the sum of guessed source matrices would be similar to the answer. When the negative peak was made after successive subtractions, the $B$ matrix was added instead of being subtracted to make the $A^{n}$ matrix similar to the zero matrix. The established reconstruction algorithm was described in Figure 2.

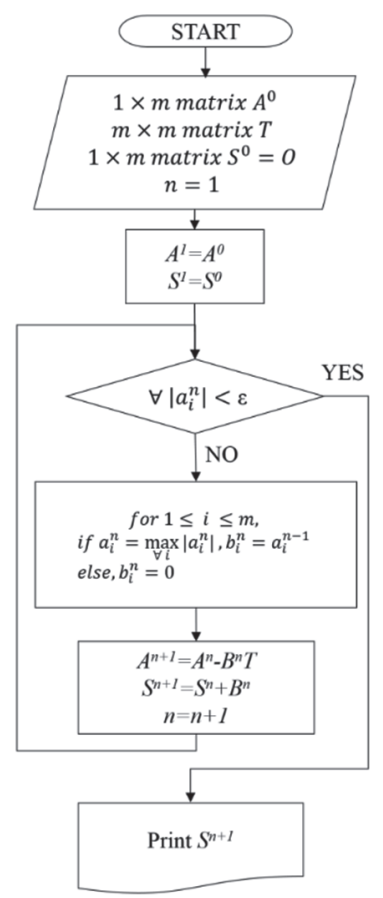

Figure 2. The flow chart of the developed reconstruction algorithm where $i$ denotes index of element of matrix and $n$ presents iteration number.

\subsection{The contribution matrix}

The contribution matrix $T$ is one of the necessary inputs to start the algorithm. The contribution matrix $T$ of the proposed measurement system was calculated by using MCNPX 2.7 code [4]. Calculated contribution $T$ was illustrated in Figure 3. The source region and the

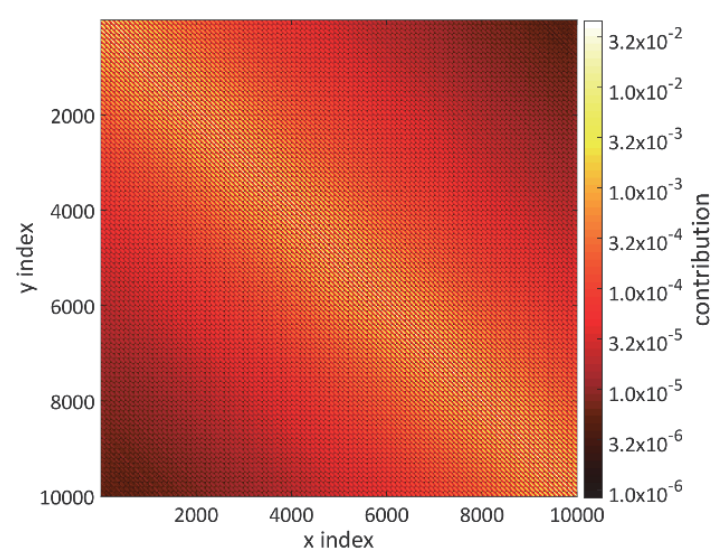

Figure 3. The contribution matrix used in the developed algorithm. 


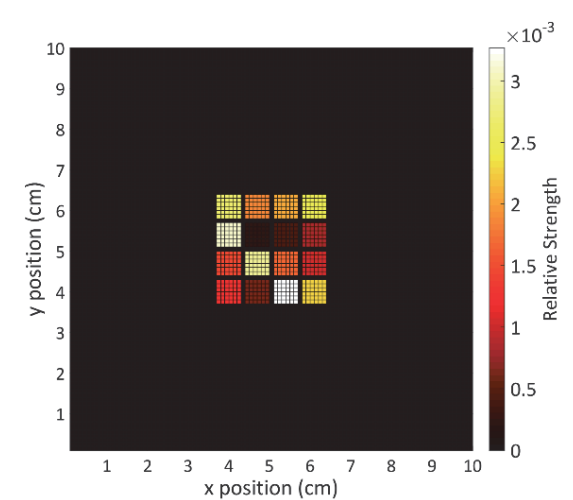

Figure 4. The array of activated foils.

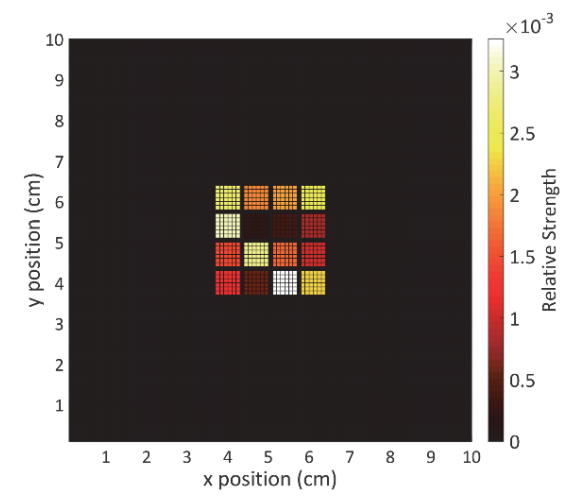

Figure 6. The reconstructed solution by the proposed algorithm.

image region were divided into 10,000 meshes each. Thus, the size of the contribution matrix $T$ was 10,000 by 10,000 . The contribution matrix was obtained with the MCNPX by simulating transport of 120,500 particles.

\section{Results and discussion}

\subsection{The reconstruction solution of the simple case}

The developed algorithm was embedded in the script of MATLAB [5]. To validate the performance of the developed algorithm, a virtual problem was made. In this problem, 16 activated foils with different strength were used. The foils were $6 \mathrm{~mm}$ by $6 \mathrm{~mm}$ square-shaped. The array of foils was 4 by 4 . The gap between foils was set to be $1 \mathrm{~mm}$, the minimum distance of resolution of this algorithm. The array of activated foils was illustrated in Figure 4. The image acquired in the CCD gamma camera was calculated using MCNPX 2.7 code and illustrated in Figure 5. In Figure 6, the reconstructed solution was illustrated. The relative error of the reconstructed solution was described in Figure 7. The relative error was calculated as the ratio of the absolute error of the calculation value to the answer.

As a result, the maximum relative error of the reconstructed solution was $1.61 \%$. The relative error was highest in the activated foil with low activity but surrounded by foils with high activity. This is because separation of weak signals from strong signals is associated with larger uncertainty. However, relative

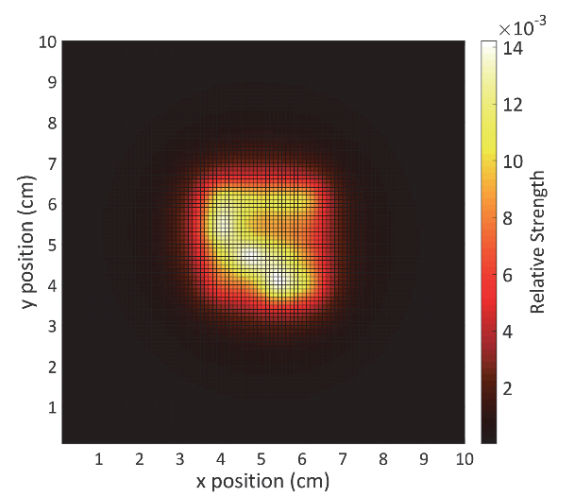

Figure 5. The image of CCD camera simulated by MCNPX.

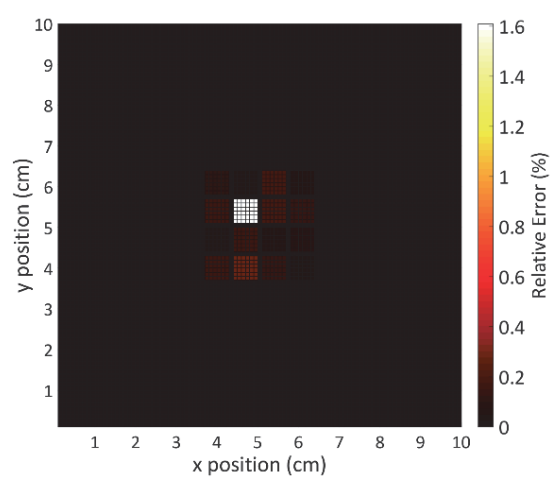

Figure 7. The error map of the reconstructed solution.

error less than $5 \%$ which is achieved in this study is acceptable to use this algorithm.

\subsection{The reconstruction of neutron flux distribution of nuclear research reactor $L V R-15$}

The reconstruction algorithm was utilized with data from the real experiments. A research to determine neutron spatial distribution in the nuclear research reactor LVR-15 was performed in Czech Republic [6]. In that research, the spatial neutron flux was measured in 16 by 16 regions. To apply those data to this study, it was assumed that the 256 activation foils were irradiated in the research reactor and measured in the proposed measurement system. In the research, the active diameter of the converter in the detector was $3 \mathrm{~mm}$. To set similar condition with the experiment, the size of foil was set to be $3 \mathrm{~mm}$ by $3 \mathrm{~mm}$. The interference of radiations from foils could be reduced by placing foils apart from one another. To place all foils with possibly broad gap in the detection system, the gap between foils was set to be $3 \mathrm{~mm}$. If the gap between foils was smaller, more interferences of radiations could be occurred. It might bring higher relative errors in the reconstruction result. The activated foils array was described in Figure 8. The image acquired in the CCD gamma camera was calculated with MCNPX and it was illustrated in Figure 9. The solutions were reconstructed by utilizing the developed algorithm. In Figure 10, the reconstructed solution was described. The relative error of the solution was illustrated in Figure 11. 


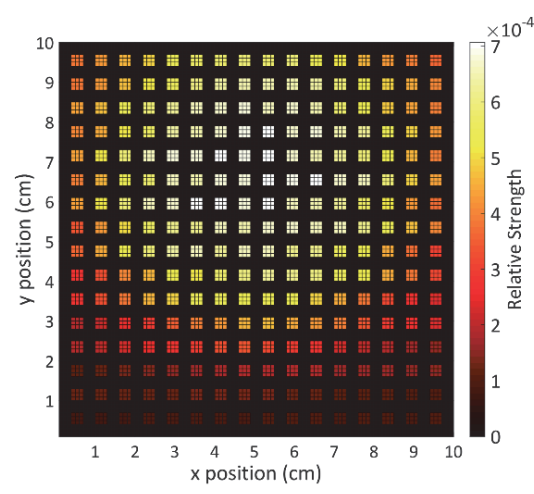

Figure 8. The array of foils assumed to be activated in the research reactor LVR-15.

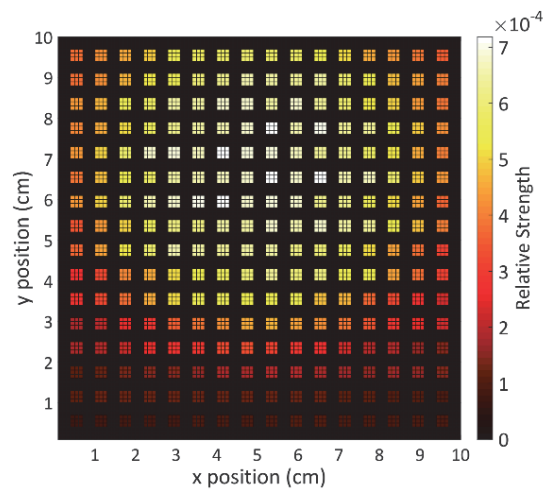

Figure 10. The reconstructed solution by the proposed algorithm.

As a result, the maximum relative error of the solution was $4.35 \%$. The maximum relative error occurred in the region with low neutron flux.

\section{Conclusion}

The purpose of this study was to develop a reconstruction algorithm and figure out its possibility of use in the proposed measurement system. To accomplish this goal, a novel reconstruction algorithm was developed. The main idea of the developed algorithm was to utilize the contribution matrix. This matrix described the transport process of radiations in the measurement system.

The developed algorithm was validated by applying to a virtual measurement system modeled by the simulation code. As a result, it was shown that the acceptable solution was reconstructed with the novel algorithm. It is expected that the developed algorithm of this study could be used for the efficient measurement of neutron beam distribution.

\section{Acknowledgements}

This work was supported by the Energy Efficiency \& Resources of the Korea Institute of Energy Technology Evaluation and Planning (KETEP) grant funded by the Korea government Ministry of Knowledge Economy (20171510300470)

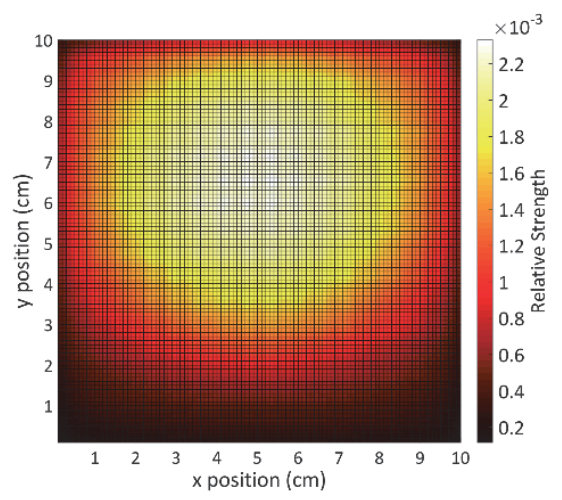

Figure 9. The image of CCD gamma camera simulated by MCNPX.

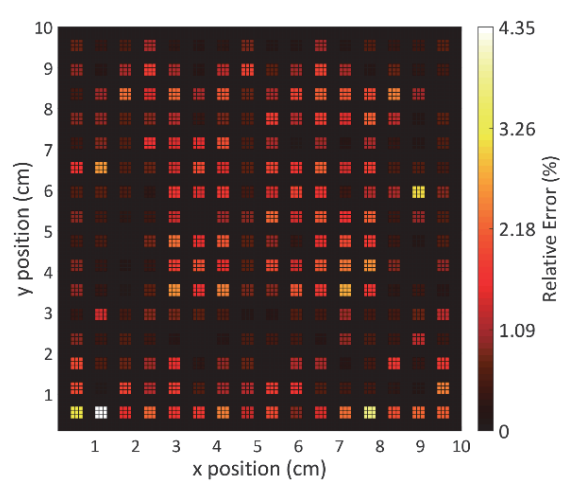

Figure 11. The error map of the reconstructed solution.

\section{References}

[1] M. Tamaki and M. Estermann, Dy-IP characterization and its application for experimental neutron radiograpy tests under realistic conditions, Nuclear Instruments and Methods in Physics Research, Section. A 542 (2005), pp.320-323.

[2] J. Kim, G.M. Sun, H. Baek and S.M.T. Hoang, Fabrication of activation foil arrays using printing techniques for evaluation of spatial distribution of neutron flux and energy, Journal of Radioanalytical and Nuclear Chemistry 307 (2016), pp. 2133-2136.

[3] NeutronOptics Manual, Retrieved from http:// http://hewat.net/nog/Manual-NeutronOptics-Imagi ng-Camera.pdf

[4] D.B. Pelowitz, MCNPX User's Manual, LA-CP-11-00438, Los Alamos National Laboratory, (2017)

[5] The Math Works, Inc., MATLAB Primer, The Math Works, Inc. (2017)

[6] M. Rabocova, M. Vins and J.Soltes, Experimental determination of neutron beam spatial distribution of nuclear research reactor LVR-15 for BNCF application, American Journal of Engineering Research 6 (2017), pp. 203-206. 